\title{
Orientation Estimation in Ambiguous Neighbourhoods
}

\author{
Mats T. Andersson \& Hans Knutsson \\ Computer Vision Laboratory Linköping University \\ 58183 Linköping, Sweden
}

\begin{abstract}
This paper describes a new algorithm for local orientation estimation. The proposed algorithm detects and separates interfering events in ambiguous neighbourhoods and produces robust estimates of the two most dominant events. A representation suitable for simultaneous representation of two orientations is introduced. The main purpose of this representation is to make averaging of outputs for neigbourhoods containing two orientations possible.

The feature extraction is performed by a set of quadrature filters. A method to obtain a large set of quadrature filter responses from a limited basis filter set is introduced. The estimation of the neighbourhood and the separation of the present events are based upon the quadrature responses in terms of local magnitude and phase. The performance of the algorithm is demonstrated using test images.
\end{abstract}

\section{Introduction}

It is widely accepted that the most powerful way to solve difficult image processing problems is to use a hierarchical processing structure. One of the most profound operations in such a scheme is the local orientation estimate for line and edge elements, which is the subject for this paper. Most algorithms for orientation estimation assume the neighbourhood to be locally one-dimensional i.e. consisting of a line or an edge. For natural images this assumption is correct in most cases. Ambiguous neighbourhoods such as corners and crosses do, however, contain important information for the interpretation of the scene at higher levels in the hierarchy.

The purpose of the proposed algorithm is to separate ambiguous neighbourhoods into several one-dimensional events that can be propagated to the next level as alternative events. In such a way the degree of freedom in the model is increased while the benefits of the one-dimensional approach is preserved. The description of the algorithm will come in two parts. First a basis filter set is introduced that enables an approximation of a quadrature filter pair in an arbitrary direction. Since the algorithm will require a dense feature extraction such a filter set is computationally effective but not necessary. The second part of the paper describes how single events can be extracted in an ambiguous environment by observing the quadrature responses in terms of magnitude and phase. For each neighbourhood the algorithm will calculate two orientation estimates. The resulting images can be averaged and offer a thorough interpretation of the neighbourhood that fits well in a hierarchical processing structure. 


\section{Feature Extraction}

To perform a more exhaustive analysis of a neighbourhood, a denser partitioning of the Fourier domain is necessary compared to models based on local one-dimensionality. The partitioning of the Fourier domain is based on quadrature filter responses over a large number of orientations. Quadrature filters can with advantage be defined in polar coordinates and the response in terms of phase and magnitude enable a continuous representation of edge and line responses. A neighbourhood of a gray scale image $i(x, y)$ has a Hermitian Fourier transform $I(\bar{\omega})=I\left(\omega_{x}, \omega_{y}\right)=I^{*}\left(-\omega_{x},-\omega_{y}\right)$ which imply that the real part $\operatorname{Re} I(\bar{\omega})$ is even and the imaginary part $\operatorname{Im} I(\bar{\omega})$ is odd. The energy contribution of the local neighbourhood $i(x, y)$ can in the Fourier domain be obtained as:

$$
|I(\bar{\omega})|^{2}=|I(-\bar{\omega})|^{2}=[\operatorname{Re} I(\bar{\omega})]^{2}+[\operatorname{Im} I(\bar{\omega})]^{2}
$$

As $|I(\bar{\omega})|^{2}$ is even, it is sufficient to estimate the energy in one halfplane, and the even and odd parts of $I(\bar{\omega})$ can be extracted separately. A general quadrature filter can be expressed as:

$$
\begin{aligned}
& H_{e}(\rho, \varphi)=\frac{1}{2} \cdot G(\rho) \cdot\left[\Gamma\left(\varphi-\varphi_{k}\right)+\Gamma\left(\varphi-\varphi_{k}+\pi\right)\right] \\
& H_{o}(\rho, \varphi)=\frac{1}{2} \cdot G(\rho) \cdot\left[\Gamma\left(\varphi-\varphi_{k}\right)-\Gamma\left(\varphi-\varphi_{k}+\pi\right)\right] \\
& H(\rho, \varphi)=H_{e}(\rho, \varphi)+H_{o}(\rho, \varphi)=G(\rho) \cdot \Gamma\left(\varphi-\varphi_{k}\right)
\end{aligned}
$$

where:

$G(\rho)$ defines the frequency characteristics of the quadrature filter, $\rho=\sqrt{\omega_{x}^{2}+\omega_{y}^{2}}$.

$\varphi_{k}$ is the main direction of the filter, $\varphi=\tan ^{-1}\left(\frac{\omega_{y}}{\omega_{x}}\right)$.

$\Gamma(\varphi)$ controls the angular performance of the filter. $\Gamma(\varphi)=0$ for $|\varphi| \geq \pi / 2$.

The aim is now to produce a large set of quadrature filter responses which can support the proposed model. To compute each of these filter responses separately would lead to heavy and redundant computations. A more attractive way is to produce a small basis filter set from which quadrature responses can be approximated in an arbitrary orientation $\left(\varphi_{k}\right)$ and with alternative angular discrimination functions $\Gamma(\varphi)$. A straightforward and robust method to accomplish this task is to produce a basis filter set, $\left[F_{e}(i), F_{o}(i)\right]$, of harmonic filters, i.e. filters that in the Fourier domain have angular functions corresponding to $\cos (i \varphi)$ and $\sin (i \varphi)$ such that:

$$
\begin{array}{ll}
F_{e}(i)=G(\rho) \cdot \cos (i \varphi) & i=[0,1 \ldots n] \\
F_{o}(i)=G(\rho) \cdot \sin (i \varphi) & i=[1,2 \ldots n]
\end{array}
$$

The angular bandwidth, $n$, of the basis filter set will determine the upper bounds for the angular resolution $\Gamma(\varphi)$. An increase of the angular bandwidth will, however, require larger convolution kernels which reduce the spatial resolution according to the principle of uncertainty for the spatial and frequency domain. The results presented in this paper were obtained from basis kernels of size $15 \times 15$ pixels and $n=6$. 
An expression for the quadrature response (Eq. 2) in an arbitrary orientation $\left(\varphi_{k}\right)$ can now be approximated by using elementary features of harmonic functions.

$$
H(\rho, \varphi)=\omega(0) F_{e}(0)+\sum_{i=1}^{n} \omega(i)\left[F_{e}(i) \cos \left(i \varphi_{k}\right)+F_{o}(i) \sin \left(i \varphi_{k}\right)\right]
$$

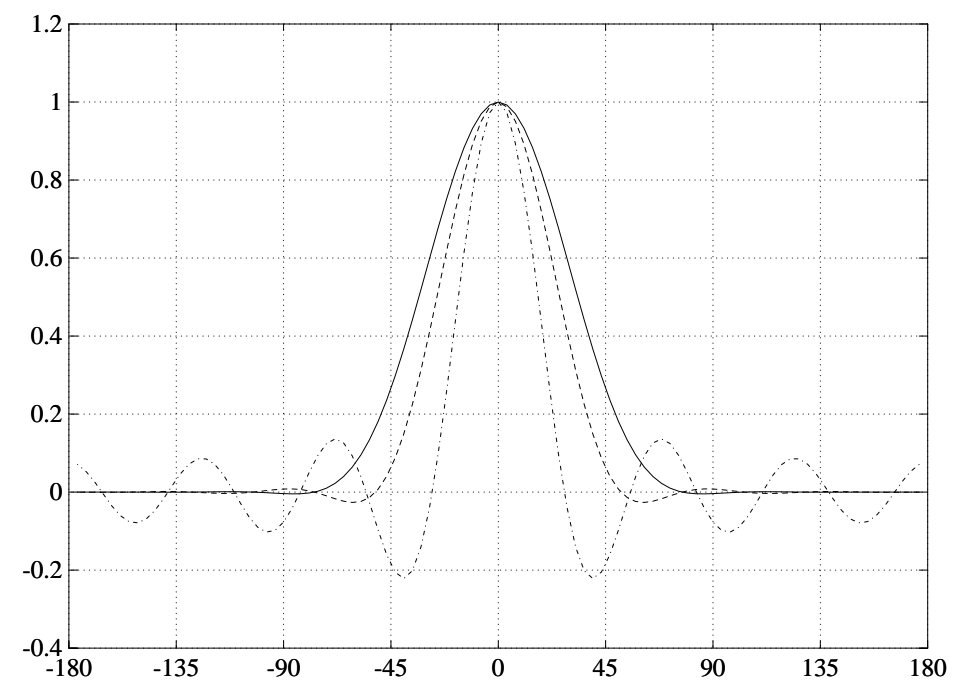

Figure 1: $\Gamma(\varphi)$ for three different weight vectors $\omega(i)$

The weight vector $\omega(i) i=[0,1 \ldots n]$ defines the $\Gamma(\varphi)$-function. Figure 1 show three normalized plots of $\Gamma(\varphi)$ corresponding to different $\omega(i)$. The DC-level is controlled by $\omega(0)$ which must be tuned to an optimal approximation of a quadrature filter. The remaining weight coefficients is in this example defined by (from wide to narrow $\Gamma(\varphi)$ ): $\omega(i)=\cos ^{4}(i / 2 n+2), \omega(i)=\cos ^{2}(i / 2 n+2)$ and $\omega(i)=1$. In all cases $n=6$. The ripple effect that may occur for narrow $\Gamma(\varphi)$ functions corresponds to a phase shift of $180^{\circ}$. As will be explained later this effect is not critical for this algorithm. To obtain $H_{e}(\rho, \varphi)$ and $H_{o}(\rho, \varphi)$ separately, equation 4 is split in terms of odd and even components of $(i)$ so that:

$$
\begin{aligned}
& H_{e}(\rho, \varphi)=\sum_{i=0,2,4 \ldots \omega(i) \cdot\left[F_{e}(i) \cos \left(i \varphi_{k}\right)+F_{o}(i) \sin \left(i \varphi_{k}\right)\right]}^{n} \omega(i) \cdot\left[F_{e}(i) \cos \left(i \varphi_{k}\right)+F_{o}(i) \sin \left(i \varphi_{k}\right)\right]
\end{aligned}
$$

The desired quadrature response can now be calculated from the convolution response between the image and the inverse Fourier transform of the basis functions. Figure 2 show a quadrature kernel pair $h_{e}(x, y)$ and $h_{o}(x, y)$ corresponding to Eq. 5. The intermediate $\Gamma$ function of Figure 1 was used. The kernel size is $15 \times 15$ and $G(\rho)$ is a lognorm function with a center frequency of $\pi / 2$ and a bandwidth of two octaves. 

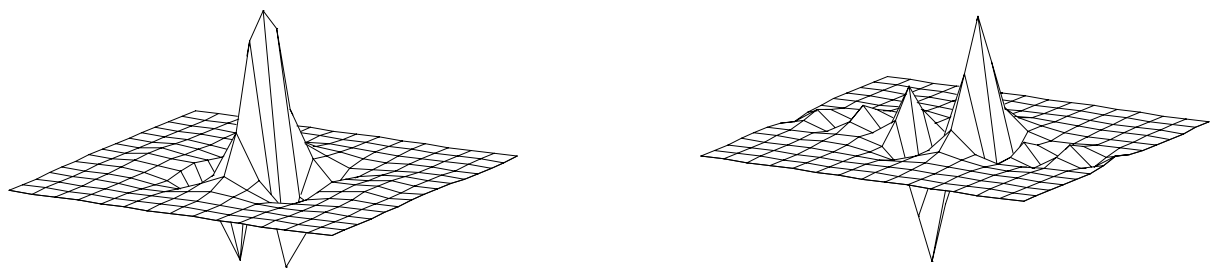

Figure 2: Wireplot of a resulting quadrature kernel $h_{e}(x, y)$ and $h_{o}(x, y)$.

From a basis set consisting of $2 n+1$ kernels it is subsequently possible to calculate quadrature responses in an arbitrary number of orientations, where $(n)$ defines the upper bounds in the trade-off between directional and spatial resolution. In this example $n=6$ results in 13 basis kernels. It may be interesting to compare this computational effort to what is required for simple one-dimensional neighbourhood. It can be shown, see [2], that the dominant orientation of a one dimensional neighbourhood can be estimated by a minimum of three quadrature filter pairs. For complex or noisy neighbourhoods the performance is considerably enhanced by four filter pairs, which results in $6-8$ kernels. The computational cost for the convolution in relation to a one dimensional approach is well below a factor of two which must be considered fairly low in comparison to the benefits gained.

\section{Estimation of Orientation in Ambiguous Neighbour- hoods}

The purpose is now to use the samples of the Fourier domain provided by the quadrature responses to estimate the present events in the neighbourhood. The ambition is that the algorithm should describe the neighbourhood in terms of one-dimensional events as far as possible. An ambiguous neighbourhood will subsequently be interpreted as several superimposed one-dimensional events, of which the two most dominant are produced as output.

To initialize the processing a number of $(N)$ quadrature filter responses, evenly distributed in one halfplane of the Fourier domain, are calculated. It may seem odd that the knowledge of the neighbourhood increases when $(N)$ increases as all responses are calculated from the same basis filter set, but as these calculations involve nonlinearities (Eq. 6) this is true to a certain extent. No qualitative investigation of the relationship between the calculated number of filter responses $(N)$ and the angular resolution of the filters $\Gamma(\varphi)$ has so far been carried out. The results in the end of this section were obtained by $N=24$. 
The quadrature response in direction $\varphi_{k}=\pi k / N \quad k=[0,1 \ldots N-1]$ is denoted $X_{k}=X_{k e}+j \cdot X_{k o}$, where $X_{k e}$ and $X_{k o}$ refer to the even and odd filter response. The magnitude $\left|X_{k}\right|$ and the Fourier phase $\theta_{k}$ are for each filter pair defined as:

$$
\left|X_{k}\right|=\sqrt{X_{k e}^{2}+X_{k o}^{2}} \quad \theta_{k}=\tan ^{-1}\left(\frac{X_{k o}}{X_{k e}}\right)
$$

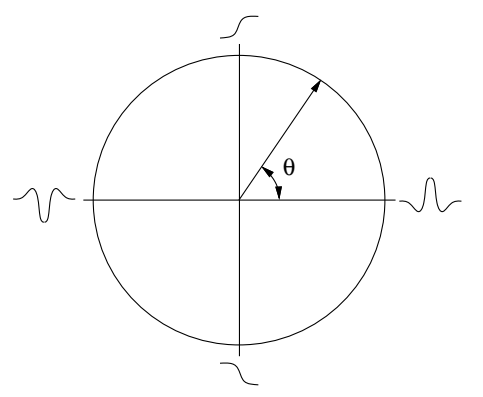

Figure 3: Continuous line/edge detection by the Fourier phase.

The Fourier phase $\theta_{k}$ can according to Figure 3 be interpretated as a shape estimate for the part of the neighbourhood that corresponds to $\Gamma\left(\varphi-\varphi_{k}\right)$. For $\theta_{k}$ equal to zero or $\pi$ the corresponding part of the neighbourhood will be interpreted as a light respectively dark line, while $\theta_{k}= \pm \pi / 2$ corresponds to edges of 'different sign'. The Fourier phase thus offers a continuous shape description of a part of a neighbourhood in terms of onedimensional events. The magnitude $\left|X_{k}\right|$, which is phase independent, reflects the energy present in the same part of the neighbourhood.

To be able to separate interfering events, a continuous description of both the shape and the dominant orientation for every part of the neighbourhood are required. The shape criterion is well met by the Fourier phase $(\theta)$, but the orientation representation require some consideration. Assume for a moment that the neighbourhood is onedimensional. This will in the Fourier domain result in a concentration of the energy upon a line in some direction $\varphi \in[0, \pi]$, and the magnitude of the corresponding quadrature responses will be high. The angle $\varphi$ is, however, ambiguous as $\varphi_{N-1}-\varphi_{0}=\pi(1-1 / N) \approx \pi$, while $\varphi_{N-1}$ and $\varphi_{0}$ correspond to practically the same orientation. To avoid this ambiguity the concept of the double angle representation is introduced, see [2]. For a one-dimensional neighbourhood the dominant orientation can be calculated as

$$
R=\sum_{k=0}^{N-1}\left|X_{k}\right| \cdot e^{j \cdot 2 \varphi_{k}}
$$

where $|R|$ corresponds to the energy in the neighbourhood and $\arg (R)$ is an unambiguous estimation of the orientation as $\varphi_{0}$ and $\varphi_{N-1}$ are mapped next to each other. What will happen if several events are present? Due to the nature of the vector summation, orthogonal events will become opposite in the proposed representation and have a tendency to cancel each other. The result will be a reduction in the magnitude $|R|$ for ambiguous events, and the estimated orientation may be expected to be an average of the present events. The last statement is, however, only true if all events obtain the same 
Fourier phase. In other cases the estimated orientation may not be relevant for either of the events due to phase interference. The optimal result for such a one-dimensional model in an ambiguous neighbourhood is thus zero, as neither the magnitude nor the estimated orientation may be relevant for any of the events. Zero output do, however, only occur for a very limited set of ambiguous neighbourhoods.
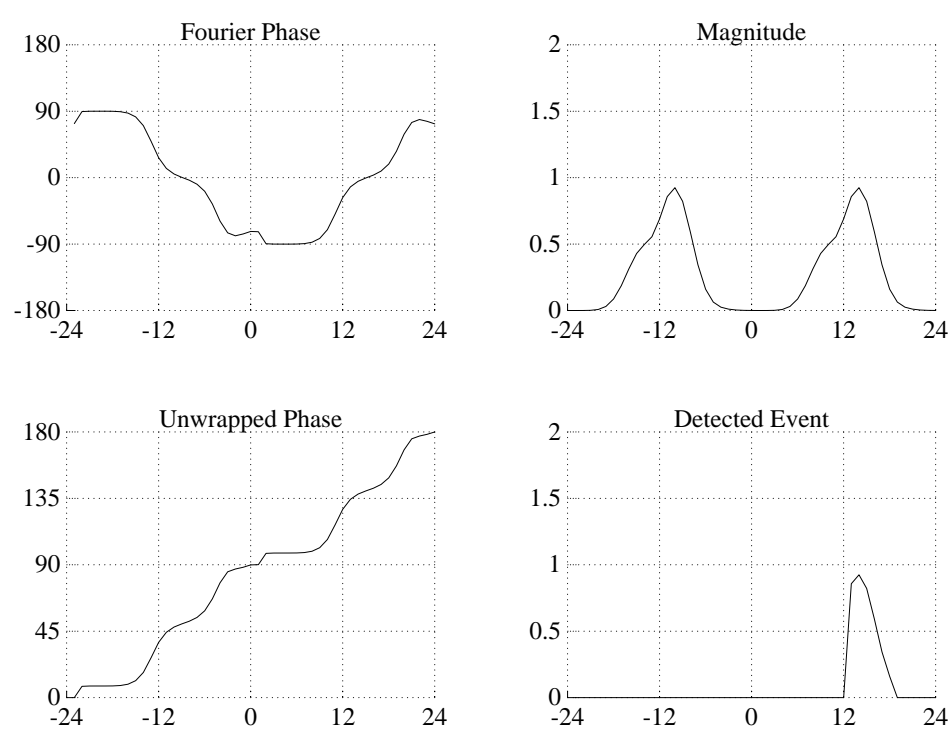

Figure 4: Illustration of phase and magnitude for an ambiguous neighbourhood.

The core of the proposed algorithm is to separate the global vector summation above into two summations as

$$
R_{a}=\sum_{k=a_{1}}^{a_{2}}\left|X_{k}\right| \cdot e^{j \cdot 2 \varphi_{k}} \quad R_{b}=\sum_{k=b_{1}}^{b_{2}}\left|X_{k}\right| \cdot e^{j \cdot 2 \varphi_{k}}
$$

where the elements of each summation correspond to a single event. Each summation is performed over a closed region in the Fourier domain and a specific magnitude $\left|X_{k}\right|$ can at most contribute to one summation. To obtain a criterion for separation of a neighbourhood in terms of phase, it may be helpful to study the magnitude and phase response of a typically ambiguous neighbourhood. The upper curves of Figure 4 show the magnitude and phase responses for a neighbourhood consisting of a cross between a line and an edge whose orientation differs by $30^{\circ}$. The magnitude of the two events differ a factor of two and there is noise present. For clarity the samples corresponding to the whole Fourier domain are illustrated although it is only necessary to perform the calculations over one halfplane. From these curves it can be deduced that the phase curve is more or less constant in the area that corresponds to the present events, while it is rather unstable between the events and in the noise. To develop this observation the unwrapped phase $\left(\Delta_{k}\right)$ is calculated as: 


$$
\Delta_{k}=\sum_{i=0}^{k}\left(\theta_{i}-\theta_{i-1}\right)^{2}
$$

In this operation care must be taken to disengage natural phase jumps of $2 \pi$ which are irrelevant. The unwrapped phase $\Delta(\varphi)$ is illustrated in the lower left part of Figure 4 . The algorithm can now be described as: chose two arbitrary orientations $\varphi_{l}$ and $\varphi_{h}$ who fulfill the condition: $\Delta\left(\varphi_{h}\right)-\Delta\left(\varphi_{l}\right) \leq \lambda$ and calculate the sum:

$$
R_{a}=\sum_{k=l}^{h}\left|X_{k}\right| \cdot e^{j \cdot 2 \varphi_{k}}
$$

This sum is calculated for all possible candidates of $\varphi_{l}$ and $\varphi_{h}$. As the first output then choose the resulting sum $R_{a}$ that obtained the largest magnitude. Note that if the $\Delta(\varphi)$-curve is flat in an environment, the summation will engage more terms and the resultant magnitude is more likely to be large. If the parameter $\lambda$ is chosen with care $R_{a}$ will correspond to the most dominant event in the neighbourhood. The part of the magnitude curve corresponding to $R_{a}$ is then disengaged and the algorithm estimates the second event, if present, by the same method. To discriminate non-relevant outputs, especially for the second event, an additional criterion may be used which demands that an event must span a certain area of the Fourier domain to be accepted. The proposed phase criterion would theoretically fail if the neighbourhood consists of two black or two white lines, as no phase shifts would then occur. In practice, however, there is always noise present both in the image and in the filters which will help the algorithm to focus on the actual events.

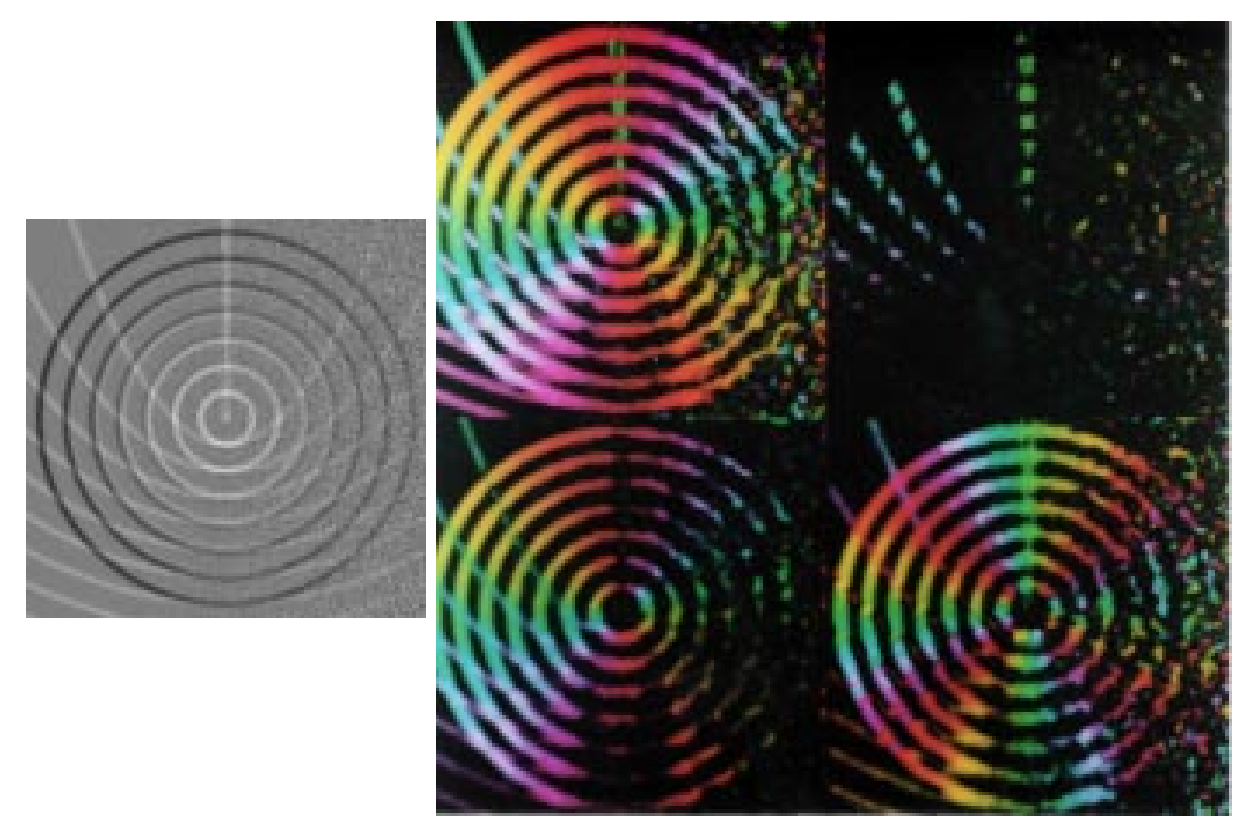

Figure 5: Test Image containing interfering events of different phase. 
Figure 5 show the results of the algorithm on a test image. The original image is displayed to the left and contain ambiguous neighbourhoods where both the relative orientation and relative phase of the events vary. On the top to the right the magnitude of two dominant events $\left|R_{a}\right|$ and $\left|R_{b}\right|$ are estimated. Note that $\left|R_{b}\right|$ is close to zero for one-dimensional neighbourhoods. The argument (orientation) of the estimated events is encoded in colour which unfortunately cannot be reproduced here.

It is naturally possible go on and estimate more than two events in many cases, as there are no built in limitations in the algorithm nor in the proposed model. It is, however, important to consider the possibility to average the results in a meaningful way. The output images on top of Figure 5 corresponding to $R_{a}$ and $R_{b}$ can not be averaged as there is no guarantee that the events may not flip between the images. For two events this problem can be solved by the following bijective transformation which permit a meaningful averaging:

$$
\left[R_{a}+R_{b}\right] \quad \text { Double angle of }\left[R_{a}-R_{b}\right]
$$

The result is displayed in the lower part of Figure 5. For more than two events the averaging must be performed in three dimensions, $(x, y, \varphi)$.

To sum up, the proposed algorithm will perform a robust and more complete estimation of a complex and noisy neighbourhood compared to earlier methods based on local one-dimensionality. The results can be averaged and fits well in a hierarchical processing structure. This concept can further on, by simple modifications be used to detect special cases of ambiguous neighbourhoods in a direct way. It is for example possible that in a one level operation detect orthogonal crosses between a light and a dark line, or to estimate the angle between two crossing lines.

\section{References}

[1] Gösta H. Granlund: In Search of a General Picture Processing Operator. Computer Graphics and Image Processing, Vol. 8, No. 2, pp 155-178, October 1978.

[2] Hans Knutson: Filtering and Reconstruction in Image Processing, Thesis No.88, Linköping University, 1982.

[3] William T. Freeman \& Edward H. Adelson: The Design and Use of Steerable Filters for Image Analysis, Enhancement, and Wavelet Representation, Technical Report 126a, Massachusetts Institute of Technology, September 1990.

[4] Pietro Perona: Deformable kernels for Early Vision, Proceedings of CVPR June 91, Maui Hawaii.

[5] Ronald N. Bracewell: The Fourier transform and Its Applications. McGraw-Hill, New York, 1978. 